\title{
IMPACT OF THERMAL TREATMENT ON THE ANTIOXIDANT ACTIVITY OF CORNELIAN CHERRIES EXTRACT
}

\section{BIANCA MOLDOVAN ${ }^{a}$, LUMINIŢA DAVID ${ }^{a *}$, SORIN CLAUDIU MAN ${ }^{b}$}

\begin{abstract}
The present study aims to investigate the influence of the temperature on the radical scavenging capacity (measured by the 2, 2azinobis (3-ethylbenzothiazolyne-6-sulphonic acid) diammonium salt radical cation assay) and ferric reducing antioxidant power (FRAP) of Cornelian cherry fruit extract. The impact of the thermal treatment was investigated by monitoring these parameters at $75^{\circ} \mathrm{C}$ and comparing the obtained values to those resulted by refrigerated storage of the extract. The Cornelian Cherry extract showed a great stability of the antioxidant activity after 10 days of storage at $75^{\circ} \mathrm{C}$ (ca. $29 \%$ loss of antioxidant capacity).
\end{abstract}

Keywords: Cornelian cherries, antioxidant activity, thermal stability

\section{INTRODUCTION}

The beneficial biological effects of plant-derived nutrients are well indicated by their antioxidant properties. Phytochemicals from fruits and vegetables attracted lately a great attention, especially on their ability in preventing oxidative stress caused diseases such as heart and neurodegenerative illnesses, cancer, arthritis [1, 2]. Cornelian cherries (Cornus mas L.) can be a valuable source of antioxidant compounds and their consumption may reduce the risk of several degenerative diseases. The main phytochemicals acting as antioxidants and free radical scavengers in these fruits are: ascorbic acid, anthocyanins, flavonoids and other polyphenols [3]. Cornelian cherries can be consumed fresh, dried or processed as marmalades or beverages.

\footnotetext{
a Babeş-Bolyai University, Faculty of Chemistry and Chemical Engineering, 11 Arany Janos Str., 400028, Cluj-Napoca, Romania

b Mother and Child Department, "Iuliu Hatieganu" University of Medicine and Pharmacy, 15 V. Babes Str., 400012, Cluj-Napoca, Romania

*Corresponding author: muntean@chem.ubbcluj.ro
} 
The most extensively used method in the food processing is thermal treatment. The microbiological stability and the extent of shelf-life can be achieved by this method. Thermal processing may affect the antioxidant capacity of foods. Some studies mentioned the decrease of this parameter where others have reported an increase of the antioxidant activity after thermal treatment $[4,5]$. The health promoting capacity of fruits is therefore affected by their processing history. Processing, especially thermal, is expected to affect the content and the biological activities of the bioactive components in fruits. Although the thermal degradation of Cornelian cherries' polyphenols was already investigated $[6,7]$ there is no study reporting the influence of the temperature on the stability of the antioxidant capacity of these fruits. There is well known that the total phenolic content is not necessarily correlated to the antioxidant activity and the thermal processing can different affect these two parameters which may vary in opposite ways. By investigating the effect of thermal treatment on cactus pears, JaramilloFlores et al. [8] reported a significant decrease in the total phenolic content at high temperatures but an increase of the antioxidant activity. This fact can be due to the presence of other antioxidant phytocompounds such as ascorbic acid and carotenoids. The reduction in phenolic content could not always fully predict the reduction in the antioxidant activity. Thus, the objective of this study was to investigate the effect of heating on the free radical scavenging effectiveness and reducing ability of the Cornelian cherry fruits extract.

\section{RESULTS AND DISCUSSION}

Cornelian cherry (Cornus mas L.) fruits are known for their elevated antioxidant activity, characteristic related to the presence of anthocyanins, flavonoids and other phenolic compounds [9]. That's why these less consumed fruits may be considered a valuable source of antioxidant compounds in the diet.

As the antioxidant capacity of fruit extracts strongly depends on the kind of individual antioxidants present in the sample, more than one assay is recommended to be used for the determination of the total antioxidant capacity of these samples. Different methods have been reported to be used for the evaluation of the in vitro antioxidant capacity of fruits of which ABTS, FRAP, DPPH, PRAP and ORAC are the most widely used [10, 11]. It is recommended that at least two of these assays to be combined in order to provide complet information on the total antioxidant activity of natural samples. FRAP (ferric reducing antioxidant power) assay and ABTS (2,2- 
azinobis(3-ethylbenzothiazolyne-6-sulphonic acid diammonium salt) assays have been lately widely applied to analyse the Trolox equivalent antioxidant capacity (TEAC) of different fruits. The determined values for the antioxidant activity were $9684.62 \mu \mathrm{M}$ Trolox and $8982.20 \mu \mathrm{M}$ Trolox in Cornelian cherries extract as determined by ABTS and FRAP assay [12] respectively. These methods are based on different mechanisms for assessing the antioxidant capacity of samples. The ABTS assay measures the free radical scavenging capacity of antioxidant compounds, while the FRAP assay indicates the ability of these compounds to act as reducing agents. The ABTS and FRAP assays use different techniques for measuring the antioxidant capacity of a sample namely the reduction capacity (FRAP) and the diradical inhibition (ABTS). The two methods offered comparable information on the measured TEAC of Cornelian cherry fruits.

Figure 1 presents changes in the antioxidant activity of the investigated extracts during storage at $2^{\circ} \mathrm{C}$ and $75^{\circ} \mathrm{C}$, measured by ABTS and FRAP assays.

ABTS assay indicated a ca. $10 \%$ reduction of the antioxidant capacity of Cornelian cherry fruits extract after 60 days of cold storage $\left(2^{\circ} \mathrm{C}\right.$, Figure 1a). The FRAP values also declined upon refrigerated storage, but the changes were higher as compared to the ABTS assay. At the end of the storage the antioxidant activity evaluated by the FRAP assay was reduced by $17 \%$.

Storage at $75^{\circ} \mathrm{C}$ (Figure $1 \mathrm{~b}$ ) resulted in a higher loss of the antioxidant capacity of the extracts. After 10 days of storage, both methods indicated a ca. $29 \%$ decrease of the antioxidant activity of the investigated extracts.
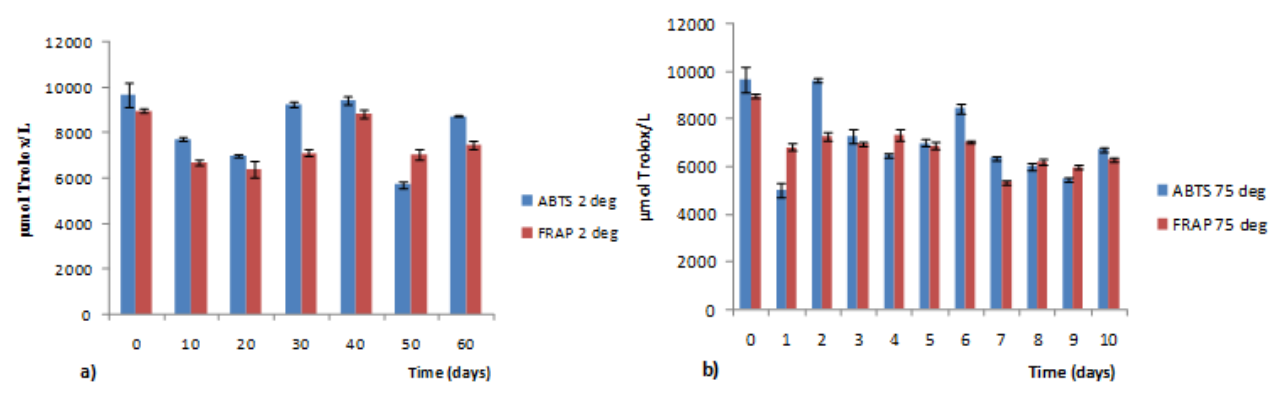

Figure 1. Changes in the antioxidant activity of the Cornelian cherries extracts during storage at: a) $2^{\circ} \mathrm{C}$ and b) $75^{\circ} \mathrm{C}$ 
Regardless of storage temperature, the Cornelian cherry fruits extracts showed good storage stability in terms of the antioxidant capacity, as evaluated by both assays. These observations are in accordance with the findings of other authors who confirmed the storage stability of antioxidant capacity of other fruits such as blueberry, apricots, pomegranate plums and raspberry [13-16]. The measured values of the antioxidant capacity strongly fluctuated during the storage periods according to both applied analytical methods. This behavior can be explained by various reactions that may occur. The reduction of the antioxidant activity may be due to degradation of water soluble phenolic compounds such as anthocyanins and vitamin $C[6,17]$. The increase of the antioxidant activity was not at all surprising, as also reported by other studies [18-20] and can be explained by compounds generated during Maillard reaction and also by formation of procyanidins, low molecular polymers with antioxidant activity or by generation of other degradation products of anthocyanins or phenolic acids, compounds which also present antioxidant capacity [21, 22].

Overall, the in vitro assays demonstrated that antioxidant capacity of Cornelian cherry fruits extract slightly decreased after the investigated storage intervals.

\section{CONCLUSIONS}

Cornelian cherry fruits are more and more investigated due to their high content of antioxidants phytocompounds which confer them numerous health benefits. The aim of the present study was to investigate the influence of temperature on the antioxidant capacity of these fruits during storage. In order to achieve this goal, the fruit extracts were stored at $75^{\circ} \mathrm{C}$ for 10 days. The decrease in the antioxidant activity observed during this storage period was not significant compared to refrigerated storage indicating that thermal treatments which are often applied in food processing and storage does not dramatically influence the health promoting antioxidant capacity of these fruits.

\section{EXPERIMENTAL SECTION}

\section{Chemicals and reagents}

All chemicals and reagents were purchased from Merck (Darmstadt, Germany), were of analytical grade and were used without further purification. A TYPDP1500 Water distiller (Techosklo LTD, Držkov, Czech Republic) was used to obtain the distilled water. 


\section{Plant material}

Samples of Cornelian cherries were purchased in August 2016 from a local market in Cluj-Napoca, Romania. Fruits of uniform ripening stage, shape and weight were selected, washed with distilled water and used to obtain the fruit extract.

\section{Extract preparation}

The extraction procedure was conducted as described in previous studies [9]. Milled Cornelian cherry fruits were subjected to acetone extraction. The extraction was conducted at room temperature for 1 hour. The solution was filtered and the filtrate was concentrated to a solvent free extract using a rotary evaporator. The extract was divided into two portions: the first was stored at $2^{\circ} \mathrm{C}$ for 60 days and the second was stored in a thermostatic water bath preheated at $75^{\circ} \mathrm{C}$, in order to investigate the effect of thermal treatment on the antioxidant activity of the samples. Changes in the antioxidant capacity of the samples were analyzed by measuring this parameter at different time intervals.

\section{Determination of antioxidant activity}

\section{ABTS Assay}

ABTS radical cation scavenging ability of the fruit extract was assessed by the method proposed by Arnao et al. [23]. Two stock solutions were prepared as follows: a $7.4 \mathrm{mM}$ ABTS and a $2.45 \mathrm{mM}$ potassium persulfate solution in distilled water. The ABTS+'was activated by mixing equal volumes of the two stock solutions which were then allowed to react for $24 \mathrm{~h}$ in the dark at room temperature. The obtained solution was diluted with distilled water to obtain an absorbance of 0.8-0.9 at $734 \mathrm{~nm}$. An amount of $100 \mu \mathrm{L}$ Cornelian cherry fruit extract (128 fold diluted) was allowed to react with $6 \mathrm{~mL}$ diluted ABTS solution for 15 minutes in the dark. Using a Perkin Elmer Lambda 25 double beam UV-Vis spectrophotometer (Perkin Elmer, Shelton, CT, USA) the absorbance of the samples and the blank was read at $734 \mathrm{~nm}$. A calibration curve was used to express the antioxidant activity of the investigated samples in $\mu \mathrm{mol}$ Trolox equivalents $/ L$ extract.

\section{FRAP Assay}

The ferric reducing antioxidant power (FRAP) assay was performed using the method of Benzie and Strain [24]. Stock solutions of $300 \mathrm{mM}$ acetate buffer ( $\mathrm{pH}=3.6), 10 \mathrm{mM}$ 2,4,6-tripyridyl-s-triazine (TPTZ) solution in 
$40 \mathrm{mM} \mathrm{HCl}$ and $20 \mathrm{mM} \mathrm{FeCl} \cdot 6 \mathrm{H}_{2} \mathrm{O}$ solution in distilled water were prepared. The working FRAP reagent was freshly prepared for each measurement of the antioxidant activity as follows: $2.5 \mathrm{~mL}$ TPTZ solution were mixed with 25 $\mathrm{mL}$ acetate buffer solution and $2.5 \mathrm{~mL} \mathrm{FeCl} \cdot 6 \mathrm{H}_{2} \mathrm{O}$ solution and the resulting mixture was warmed to $37^{\circ} \mathrm{C}$ before use. A total of $150 \mu \mathrm{L}$ fruit extract was allowed to react to $2850 \mu \mathrm{L}$ warm FRAP reagent for 30 minutes in the dark. Absorbance reading of the resulted colored complex was taken at $593 \mathrm{~nm}$ against a blank sample. The results were expressed in $\mu \mathrm{mol}$ Trolox equivalents/L extract, using a standard curve.

\section{ACKNOWLEDGMENTS}

The Ministry of Education and Scientific Research, Romania, supported this work (research Project no. 147/2011 PN-II-PT-PCCA-2011-3-1-0914).

\section{REFERENCES}

1. J. Bloomberg, Horticultural Science, 2003, 38, 771.

2. B. Moldovan, L. David, M. Achim, S. Clichici, G.A. Filip, Journal of Molecular Liquids, 2016, 221, 271.

3. L. David, B. Moldovan, Extraction, characterization and potential health benefits of bioactive compounds from selected Cornus fruits, in: Fruits and pomace extracts: biological activity, potential applications and beneficial health effects. Nova Science Publishers, Inc. New-York, USA, 2015, 157-188.

4. A. Patras, N.P. Bronton, S. Da Pieve, F. Butler, G. Downey, Inovative Food Science \& Emerging Technologies, 2009, 10, 16.

5. E. Sikora, E. Cieslik,T. Leszczynska, A. Filipiak-Florkiewicz, M. PisulewskiPawel, Food Chemistry, 2008, 107, 55.

6. B. Moldovan, A. Popa, L. David, Journal of Applied Botany and Food Quality, 2016, 89, 208.

7. B. Moldovan, L. David, Molecules, 2014, 19, 8177.

8. M.E. Jaramillo-Flores, L. Gonzalez-Cruz, M. Cornejo-Mazon, L. DorantesAlvarez, G.F. Gutierrez-Lopez, H. Hernandez-Sanchez, Food Science and Technology International, 2003, 9, 271.

9. B. Moldovan, A. Filip, S. Clichici, R. Suharoschi, P. Bolfa, L. David, Journal of Functional Foods, 2016, 26, 77.

10. B.M. Popovic, D. Stajner, K. Slavko, B. Sandra, Food Chemistry, 2012, 134, 734.

11. J. Contreras-Calderon, L. Calderon-Jaines, E. Guerra-Hernandez, B. GarciaVillanova, Food Research International, 2011, 44, 2047. 
12. A. Hosu, C. Cimpoiu, L. David, B. Moldovan, Journal of Analytical Methods in Chemistry, 2016, Article ID 2345375, 5 pages. http://dx.doi.org/10.1152016/ 2345375.

13. J.A. Hernández-Herrero, M.J. Frutos, International Journal of Food Science \& Technolog., 2011, 46, 2550.

14. J. Piljac-Zegarac, D. Samec, Food Research International, 2011, 44, 345.

15. J.A., Hernández-Herrero, M.J. Frutos, Food Chemistry, 2014, 154, 199.

16. O.A. Fawole, U.L. Opara, Industrial Crops and Products, 2013, 47, 300.

17. B. Moldovan, L. David, R. Donca, C. Chisbora, Studia UBB Chemia, 2011, 56(2), 189.

18. L.F. Amaro, M.T. Soares, C. Pinho, I.F. Almeida, O. Pinho, I.M.P.L.V.O. Ferreira, International Journal of Food Science \& Technology, 2013, 48, 2071.

19. B. Moldovan, O. Ghic, L. David, C. Chişbora, Revista de Chimie, 2012, 63, 463.

20. D. Fracassetti, C. Del Bo, P. Simonetti, C. Gardana, D. Klinis-Zacas, S. Ciappellano, Journal of Agricultural and Food Chemistry, 2013, 61, 2999.

21. L.L. Howard, R.L. Prior, R. Liyanage, J.O. Lay, Journal of Agricultural and Food Chemistry, 2012, 60, 6678.

22. C. Oliveira, L F. Amaro, O. Pinho, I.M. Ferreira, Journal of Agricultural and Food Chemistry, 2010, 58, 9006.

23. M.B. Arnao, A. Cano, M. Acosta, Food Chemistry, 2001, 73, 239.

24. I.F.F. Benzie, J.J. Strain, Analytical Biochemistry, 1996, 239, 70. 